\title{
ON THE LOWER TAIL PROBABILITIES OF SOME RANDOM SERIES ${ }^{1}$
}

\author{
BY M. A. LIFSHITS
}

Mancomtech Center, St. Petersburg and Université L. Pasteur

The behavior of tail probabilities $\mathbf{P}\{S \leq r\}, r \rightarrow 0$ is investigated, where $S$ is a series $S=\sum \lambda_{j} Z_{j}$ generated by some sequence of positive numbers $\left\{\lambda_{j}\right\}$ and by a sequence $\left\{Z_{j}\right\}$ of independent copies of a positive random variable $Z$.

We present the exact asymptotic expression for $\mathbf{P}\{\mathrm{S} \leq \mathrm{r}\}$ by means of Laplace transform $\Lambda(\gamma)=\mathbf{E} \exp \{-\gamma \mathrm{S}\}$ under weak assumptions on the behavior of the tail probabilities of $Z$ in the vicinity of zero. The bounds of accuracy are also given, and under weak supplementary smoothness conditions the asymptotic properties of the density of $\mathrm{S}$ are investigated.

1. Introduction. The main subject of our interest is the behavior of the tail probabilities

(1.1)

where $\mathrm{S}$ is a series

$$
\mathbf{P}\{\mathrm{S} \leq \mathrm{r}\}, \quad \mathrm{r} \rightarrow 0
$$

$$
\mathrm{S}=\sum \lambda_{\mathrm{j}} \mathrm{Z}_{\mathrm{j}}
$$

generated by some nonincreasing sequence of positive numbers $\left\{\lambda_{\mathrm{j}}\right\}, \sum \lambda_{\mathrm{j}}<\infty$ and by a sequence $\left\{Z_{\mathrm{j}}\right\}$ of independent copies of positive random variable $Z$ with finite variance and absolutely continuous distribution. We present the exact asymptotic expression for (1.2) by means of Laplace transform $\Lambda(\gamma)=$ $\mathbf{E} \exp \{-\gamma \mathrm{S}\}$ under mild polynomial assumptions on the tail probabilities of $Z$ in the vicinity of zero.

The history of the investigation of the tail probabilities in (1.1) is curious. The question of this kind appeared initially in the theory of Gaussian vectors in Hilbert space. Indeed, let $\chi$ be a standard normal variable and $Z=\chi^{2}$. Then, $\mathbf{P}\{\mathrm{S} \leq \mathrm{r}\}$ represents the probability of the hitting in the ball of radius $r^{1 / 2}$ for Hilbert space-valued centered Gaussian random vector such that $\left\{\lambda_{\mathrm{j}}\right\}$ coincides with the spectrum of the corresponding covariance operator. It was G. N. Sytaya [14] who found the asymptotics of (1.1) for the Gaussian case. Her result was rediscovered in [7] and [18] (cf. also [5] and [13]), where further estimates of Gaussian probabilities of small balls in Hilbert space were proposed. Moreover, in [6], [9] and [11], the noncentered balls were treated, and in [10] and [19] the Gaussian measure of $I_{p}$-balls was considered. All these works use difficult complex integration procedures. Therefore,

\footnotetext{
Received May 1995; revised April 1996.

${ }^{1}$ Research supported by International Science Foundation and Russian Foundation of Basic Research.

AMS 1991 subject classifications. Primary 60F 10; secondary 60G15.

Key words and phrases. Small balls, lower tail probabilities, Laplace transform, central limit theorem, sums of independent variables.
} 
the probabilistic background of the correspondent results remains partially obscured. An alternative approach appeared recently in [8] for general Gaussian settings. It is based on the relation of the probabilities of small balls and the entropy numbers of the unit ball of the kernel of Gaussian measure. This approach looks very robust but it is not precise enough to provide exact bounds. On the other hand, some general results and surprising examples were discovered for the Gaussian case in [12] for $\mathbf{P}\{S>r\}, r \rightarrow \infty$, by means of a kind of Laplace transform.

Several essential concepts were introduced by R. Davis and S. Resnick in [4]. First, they started to investigate (1.1) for the general case, without any relation to Gaussian specifics. Second, they observed a deep connection between the asymptotics of (1.1) and the central limit theorem (CLT) for sums of nonidentically distributed random variables. (Though we also discovered the role of CLT before being informed about the existence of [4], we acknowledge the priority of the mentioned article.) Third, Davis and Resnick noticed that the CLT mechanism works well for the case of polynomial tails of the distribution of Z. Unfortunately, apart from the indispensable polynomial restriction, a supplementary condition is also imposed in [4] on the density of $Z$, and this one turns out to be so restrictive that the Gaussian case falls out of the frame of [4]. Meanwhile, the ideas related to the CLT arrived also in Gaussian context; see [1], [5].

This situation determines our goal. We aim to obtain a version of the Davis-Resnick theorem, which contains the Sytaya Gaussian result (as well as the Gaussian result from [19]) as a particular case. Moreover, the polynomial restrictions on $Z$, that we assume to be fulfilled, are essentially weaker than their counterparts from [4]. In particular, regular variation conditions are not supposed to take place. We are also able to avoid any assumption on the spectral sequence $\lambda$ and on the density of $Z$. Under mild smoothness assumptions, the asymptotic behavior of the density of $S$ at zero is investigated. We also give the bounds of accuracy for the proposed asymptotics. It turns out that the accuracy rate depends on a polynomial index related to lower tails of $Z$. If the tails are too thin, the rate may be worse than one could expect from general CLT heuristics.

Our approach is mainly based on (a small part of) the powerful and transparent CLT technique developed recently by A. Yu. Zaitsev for other purposes (see Section 5 below). The application of this technique looks promising; also it is far outside the scope treated here. One could apply the same ideas to the case of nonidentically distributed variables, consider parametric schemes like $\lambda_{\mathrm{j}}=\lambda_{\mathrm{j}}(\beta)$ with some external parameter $\beta$, and so on. For example, we give an extension of the basic result from [9] and [11] concerning the Gaussian probabilities of strongly shifted small balls in Hilbert space.

2. Notation. Denote $F$ and $f$ respectively the distribution function and the density of $\mathbf{Z}$. Let the Laplace transform of $\mathbf{S}$ be defined as

$$
\Lambda(\gamma)=\mathbf{E} \exp \{-\gamma \mathbf{S}\} .
$$


Throughout the following, current constants depending only on $F$ are denoted by $C_{1}, C_{2}, \ldots$. Notations $\theta, \theta_{1}, \ldots$ symbolize different real or complex remainder terms, always not exceeding one in absolute value. For any random variable $Y$, we denote $L(Y)$ the distribution of $Y, Y^{0}=Y-E Y$ and $\hat{Y}(\mathrm{v})=\mathbf{E} \exp \{$ ivY $\}$. We write $X=L Y$ iff $L(X)=L(Y)$.

For any nonnegative r.v. $\mathrm{Y}$ with distribution $\mathrm{U}(\mathrm{dr})$ and $\gamma>0$, let $(\mathrm{Y}) \gamma$ denote a random variable with distribution $\exp \{-\gamma r\} U(d r) / E \exp \{-\gamma Y\}$. The corresponding operation in the space of distributions is sometimes referred to as the Esscher transform. It is widely used in the theory of large deviations for sums of random variables. All the sums of r.v. will be considered as the sums of independent r.v. In particular, we need the following objects for the investigation of (1.1) (notice that the index $\gamma$ in their notation is suppressed for simplicity):

$$
\begin{gathered}
\eta_{\mathrm{j}}=\left(\lambda_{\mathrm{j}} \mathrm{Z}_{\mathrm{j}}\right)_{\gamma}, \quad \sigma_{\mathrm{j}}^{2}=\operatorname{Var}\left(\eta_{\mathrm{j}}\right), \\
\zeta=\sum \eta_{\mathrm{j}}=\sum\left(\lambda_{\mathrm{j}} \mathrm{Z}_{\mathrm{j}}\right)_{\gamma}=\left(\sum \lambda_{\mathrm{j}} \mathrm{Z}_{\mathrm{j}}\right)_{\gamma}=(\mathrm{S})_{\gamma}, \\
\sigma^{2}=\operatorname{Var}(\zeta)=\sum \sigma_{\mathrm{j}}{ }^{2} .
\end{gathered}
$$

We will need a smoothing procedure. To this aim, we fix some auxiliary r.v. $\xi$ with symmetric infinitely differentiable density concentrated on $[-1,1]$. One could take, for example, $\xi=(2 / \pi) \arctan (g)$, with $L(g)=N(0,1)$.

Let $p, q, q_{0}, q_{0 s}$ denote the densities of r.v. $S, \zeta, \zeta^{0} / \sigma$ and $\zeta^{0} / \sigma+$ $(\varepsilon \xi / \gamma \sigma)$, respectively, where index zero stands for centering and index $\mathrm{s}$ denotes smoothing (smoothing parameter $\varepsilon$ will be specified later).

3. Polynomial conditions and main results. The following two conditions provide the polynomial behavior of the lower tail probabilities for $Z$. The first condition gives an inferior bound for the tail probability, the second one gives a superior bound. We assume that there exist $b \in(0,1), c_{1}>1, c_{2} \in$ $(0,1), c_{3}>0$ such that for each $r<c_{3}$,

$$
F(r) \leq c_{1} F(b r)
$$

and

$$
\mathrm{F}(\mathrm{br}) \leq \mathrm{c}_{2} \mathrm{~F}(\mathrm{r}) \text {. }
$$

The validity of both conditions is completely determined by the behavior of $\mathrm{F}$ in any vicinity of 0 . Moreover, it follows easily from $(*)$ and $(* *)$ that for $r \leq c_{3}$ inequalities,

hold with

$$
C_{4} r^{\alpha_{1}} \leq F(r) \leq C_{5} r^{\alpha_{2}}
$$

$$
\alpha_{1}=\log \mathrm{c}_{1} /|\log \mathrm{b}|
$$

and $\alpha_{2}=\log c_{2} / \log b, c_{4}=F\left(c_{3}\right) /\left(b / c_{3}\right)^{\alpha_{1}}, c_{5}=F\left(c_{3}\right) /\left(b c_{3}\right)^{\alpha_{2}}$. Thus, the conditions $(*),(* *)$ imply a polynomial lower tail behavior of the distribution of $\mathrm{Z}$, although they do not provide its regularity. On the other hand, $(*),(* *)$ can be verified immediately if $f(r) \sim r^{\alpha} h(r)$, where $h$ is a slowly varying 
function and $\alpha>-1$. This situation was considered in [4]. It concerns, of course, the Gaussian case $Z=|\chi|^{p}, L(\chi)=N(0,1), p>0$ where

$$
f(r) \sim(2 / \pi)^{1 / 2} p^{-1} r^{1 / p-1} .
$$

Moreover, if $F(r)=r^{\alpha} h(r)$ with $h$ slowly varying at zero, then the parameter $\alpha_{1}$ can be chosen arbitrarily close to $\alpha$. This remark is significant in view of the subsequent appearance of $\alpha_{1}$ in our accuracy rates.

Our first result is rather technical. It provides a local CLT for the smoothed convolution of the transformed terms from (1.1).

THEOREM 1. Let the conditions $(*)$ and $(* *)$ hold for the distribution $\mathrm{F}$ and $\alpha_{1}$ be defined in (3.1). Let $\zeta$ and $\sigma$ be defined in (2.1) for each $\gamma>0$. Let $\kappa \in(0,2)$ and $\varepsilon=\varepsilon(\gamma)=(\gamma \sigma)^{-(2-\kappa) / \alpha_{1}}$. Then, when $\gamma$ tends to infinity, the following hold.

(a) The distributions of $\zeta^{0} / \sigma$ converge weakly to $\mathrm{N}(0,1)$.

(b) Thedistribution densities of smoothed random variables $\left(\zeta^{0}+\varepsilon \xi / \gamma\right) / \sigma$ converge uniformly on $\mathbf{R}$ to the distribution density of $N(0,1)$, namely

$$
\sup _{u}\left|q_{0 s}(u)-(2 \pi)^{-1 / 2} \exp \left\{-u^{2} / 2\right\}\right| \rightarrow 0 \text {. }
$$

Moreover, if $\mathbf{E} Z^{3}<\infty$, then

$$
\sup _{u}\left|q_{0 s}(u)-(2 \pi)^{-1 / 2} \exp \left\{-u^{2} / 2\right\}\right|=O\left((\gamma \sigma)^{-1}\right) \text {. }
$$

The next theorem represents the main result of the article. It provides the exact asymptotic expression for tail probabilities with sharp estimate of its accuracy.

THEOREM 2. Let $S$ be the sum of series (1.2) generated by r.v. $Z$, which distribution function obeys $(*)$ and $(* *)$. Let the parameter $\gamma=\gamma(r)$ be chosen from the equation

$$
r=\mathbf{E}(\mathrm{S})_{\gamma}
$$

Then,

$$
\mathbf{P}\{\mathrm{S} \leq \mathrm{r}\}=(2 \pi)^{-1 / 2}(\gamma \sigma)^{-1} \exp \{\gamma r\} \Lambda(\gamma)[1+o(1)], \quad \mathrm{r} \rightarrow 0 .
$$

Moreover, if $\mathbf{E} Z^{3}<\infty$, then for $\alpha_{1}$ defined in (3.1) and each $\kappa \in(0,2)$,

$$
\begin{aligned}
\mathbf{P}\{\mathrm{S} \leq \mathrm{r}\}= & (2 \pi)^{-1 / 2}(\gamma \sigma)^{-1} \exp \{\gamma \mathrm{r}\} \\
& \times \Lambda(\gamma)\left[1+\mathrm{O}\left((\gamma \sigma)^{-1}+(\gamma \sigma)^{-(2-\kappa) / \alpha_{1}}\right)\right], \quad \mathrm{r} \rightarrow 0
\end{aligned}
$$


Comments. (i) According to the definition (1.2) of $\mathrm{S}$, our basic function $\Lambda$ and its derivatives can be expressed in terms of $Z$ :

$$
\begin{gathered}
\Lambda(\gamma)=\prod \mathbf{E} \exp \left\{-\gamma \lambda_{\mathrm{j}} \mathrm{Z}\right\}, \\
-(\log \Lambda)^{\prime}(\gamma)=\sum \frac{\mathbf{E} \lambda_{\mathrm{j}} \mathrm{Z} \exp \left\{-\gamma \lambda_{\mathrm{j}} \mathrm{Z}\right\}}{\mathbf{E} \exp \left\{-\gamma \lambda_{\mathrm{j}} \mathrm{Z}\right\}}=\sum \mathbf{E} \eta_{\mathrm{j}}=\mathbf{E} \zeta=\mathbf{E}(\mathrm{S})_{\gamma}, \\
(\log \Lambda)^{\prime \prime}(\gamma)=\sum\left[\frac{\mathbf{E}\left(\lambda_{\mathrm{j}} \mathrm{Z}\right)^{2} \exp \left\{-\gamma \lambda_{\mathrm{j}} \mathrm{Z}\right\}}{\mathbf{E} \exp \left\{-\gamma \lambda_{\mathrm{j}} \mathrm{Z}\right\}}-\left(\frac{\mathbf{E} \lambda_{\mathrm{j}} \mathrm{Z} \exp \left\{-\gamma \lambda_{\mathrm{j}} \mathrm{Z}\right\}}{\mathbf{E} \exp \left\{-\gamma \lambda_{\mathrm{j}} \mathrm{Z}\right\}}\right)^{2}\right] \\
=\sum \operatorname{Var}\left(\eta_{\mathrm{j}}\right)=\operatorname{Var}(\zeta)=\sigma^{2} .
\end{gathered}
$$

These representations can be effectively used for advanced analysis of (3.3) and (3.4) in many specific cases; see, for example, [18] for corresponding results and examples of asymptotics of Gaussian small ball probabilities.

(ii) It follows from the just-mentioned identities that both right-hand sides of (3.3) and (3.4) are explicitly defined by means of Laplace transform $\Lambda$ and we really have a Laplace description for asymptotics of tail probabilities.

(iii) The statement of Theorem 2 remains true if the parameter $\gamma$ is only an approximate solution of (3.3), that is,

$$
\lim _{r \rightarrow 0}\left(r-\mathbf{E}(S)_{\gamma}\right) / \sigma=0 .
$$

See the precise statement below in (6.19).

(iv) We did not impose in Theorem 2 any assumptions either on the density $f$, or on the sequence $\lambda$, or on Laplace transforms (in contrast to previous works). Our assumptions are explicitly related to the lower tails of d.f. $F$.

(v) If $\alpha_{1}<2$, then choosing $\kappa$ sufficiently small, we obtain in (3.4a) the expected accuracy rate $\left[1+\mathrm{O}\left((\gamma \sigma)^{-1}\right)\right]$. It is the same case, for example, as in the Hilbert-Gaussian situation where $\alpha_{1}=1 / 2$. It may look surprising but for thin polynomial lower tails $\alpha_{1}>2$, the accuracy rate seems to be of bad order $\left[1+\mathrm{O}\left((\gamma \sigma)^{-2-\kappa) / \alpha_{1}}\right)\right]$, at least for r.v. Z with sufficiently irregular density.

The following result describes the asymptotic behavior of the density of S.

Theorem 3. Let $S$ be the sum of series (1.2) generated by r.v. Z, which distribution function $\mathrm{F}$ obeys $(*)$ and $(* *)$. Moreover, assume that there exist some positive $C, \delta, K$ and $p>1$ such that the density $f$ of $F$ satisfies

$$
\begin{aligned}
&|f(y)-f(x)| \leq C F(x) x^{-2}(y-x), \\
& \quad \forall x, y: 0 \leq x \leq y \wedge K, 0 \leq y-x \leq \delta
\end{aligned}
$$

and

$$
\int_{K}^{\infty} f(x)^{p} d x<\infty
$$


Let $\zeta$ and $\sigma$ be defined in (2.1). Then

(a) the distribution densities $q_{0}(\cdot)$ of $r . v . \zeta^{0} / \sigma$ converge uniformly to the standard normal density.

(b) If $\gamma=\gamma(r)$ is chosen from equation (3.3), the distribution density $p(\cdot)$ of r.v. S admits an asymptotic representation

$$
\mathrm{p}(\mathrm{r})=(2 \pi)^{-1 / 2} \sigma^{-1} \exp \{\gamma r\} \Lambda(\gamma)(1+\mathrm{o}(1)), \quad \mathrm{r} \rightarrow 0 .
$$

Our technique turns out to be flexible enough to work also in the more sophisticated situation with S dependent on external parameter. We mention here only one result in this direction. This result concerns Gaussian measures. It is interesting to compare it with the corresponding recent results obtained by different methods in [9] and [11].

Let $H$ be a separable Hilbert space and let $X$ be an infinite-dimensional $\mathrm{H}$-valued centered Gaussian random vector. Then there exist an orthonormal base $\left\{e_{\mathrm{j}}\right\} \subset \mathrm{H}$, a positive sequence $\left\{\lambda_{\mathrm{j}}\right\}$ and an i.i.d. standard normal sequence $\left\{\xi_{\mathrm{j}}\right\}$ such that $\mathrm{X}=\sum \lambda_{\mathrm{j}}^{1 / 2} \xi_{\mathrm{j}} \mathrm{e}_{\mathrm{j}}$.

Let $\mathrm{a}(\mathrm{t})=\sum \alpha_{\mathrm{j}}(\mathrm{t}) \mathrm{e}_{\mathrm{j}} \in \mathrm{H}$ and $\mathrm{R}(\mathrm{t})>0$ be defined on some interval $[\mathrm{T}, \infty)$. We are interested in the asymptotic estimation of the probabilities of noncentered balls:

$$
\mathbf{P}\left\{\|\mathrm{X}-\mathrm{a}(\mathrm{t})\|^{2} \leq \mathrm{R}(\mathrm{t})\right\}=\mathbf{P}\left\{\sum\left(\lambda_{\mathrm{j}}^{1 / 2} \xi_{\mathrm{j}}-\alpha_{\mathrm{j}}(\mathrm{t})\right)^{2} \leq \mathrm{R}(\mathrm{t})\right\}, \quad \mathrm{t} \rightarrow \infty .
$$

The main ingredient of the corresponding asymptotic expression is again the Laplace transform,

$$
\begin{aligned}
\Lambda(\gamma, \mathrm{t}) & =\mathbf{E} \exp \left\{-\gamma\|\mathrm{X}-\mathrm{a}(\mathrm{t})\|^{2}\right\} \\
& =\prod\left(1+2 \gamma \lambda_{\mathrm{j}}\right)^{-1 / 2} \exp \left\{-\alpha_{\mathrm{j}}^{2} \gamma /\left(1+2 \gamma \lambda_{\mathrm{j}}\right)\right\} .
\end{aligned}
$$

The asymptotic expression, as in the previous cases, involves an additional parameter $\gamma=\gamma(\mathrm{t})$. For any $\gamma>0, \mathrm{t}>\mathrm{T}$, we define

$$
\begin{gathered}
\rho(\mathrm{t}, \mathrm{y})=\sum\left[\frac{\lambda_{\mathrm{j}}}{1+2 \gamma \lambda_{\mathrm{j}}}+\frac{\alpha_{\mathrm{j}}(\mathrm{t})^{2}}{\left(1+2 \gamma \lambda_{\mathrm{j}}\right)^{2}}\right], \\
\sigma(\mathrm{t}, \gamma)^{2}=\sum\left[\frac{2 \lambda_{\mathrm{j}}^{2}}{\left(1+2 \gamma \lambda_{\mathrm{j}}\right)^{2}}+\frac{4 \lambda_{\mathrm{j}} \alpha_{\mathrm{j}}(\mathrm{t})^{2}}{\left(1+2 \lambda_{\mathrm{j}}\right)^{3}}\right] .
\end{gathered}
$$

THEOREM 4. Assume that a choice of the parameters $\gamma=\gamma(\mathrm{t})$ provides that the relations

$$
\lim _{t \rightarrow \infty} \gamma \sigma(t, \gamma)=\infty
$$

and

$$
\lim _{t \rightarrow \infty}(\mathrm{R}(\mathrm{t})-\rho(\mathrm{t}, \gamma)) / \sigma(\mathrm{t}, \gamma)=0
$$


are satisfied. Then

$$
\begin{aligned}
\mathbf{P}\left\{\|\mathrm{X}-\mathrm{a}(\mathrm{t})\|^{2} \leq \mathrm{R}(\mathrm{t})\right\}= & (2 \pi)^{-1 / 2}(\gamma \sigma(\mathrm{t}, \gamma))^{-1} \\
& \times \exp \{\gamma \mathrm{R}(\mathrm{g})\} \Lambda(\gamma)(1+\mathrm{o}(1)) .
\end{aligned}
$$

This theorem is a slight generalization of a result due to W. V. Li and W. Linde [11], who considered the particular case $\alpha_{\mathrm{j}}(\mathrm{t})=\mathrm{f}(\mathrm{t}) \alpha_{\mathrm{j}}(0)$, that is, the centers of the balls are supposed to be located on a line; compare also a previous version of the same result in [9].

If we put $\mathrm{S}=\mathrm{S}(\mathrm{t})=\|\mathrm{X}-\mathrm{a}(\mathrm{t})\|^{2}$, it is easy to calculate that $\rho(\mathrm{t}, \gamma)=\mathbf{E}(\mathrm{S})_{\gamma}$ and $\sigma(\mathrm{t}, \gamma)^{2}=\operatorname{Var}(\mathrm{S})_{\gamma}$. Therefore, we observe that (3.9) is a version of (3.5). The assumption (3.8) is also natural and cannot be omitted. Moreover, the quantity $(\gamma \sigma)^{-1}$ naturally appears in the accuracy rate of the presented asymptotics.

The next two sections provide the technical background for the proofs of our theorems given in Section 6.

4. Properties of exponential families. Some useful properties of two families of random variables, $X_{1}=\left\{\gamma(Z)_{\gamma}, \gamma \geq 1\right\}$ and $X_{2}=\left\{(Z)_{\gamma}, 0 \leq \gamma \leq 1\right\}$ will be established now. In view of the definition of $(Z)_{\gamma}$, it is natural to call $X_{1}$ and $X_{2}$ exponential families. We will show that both families $X_{1}$ and $X_{2}$ are uniformly bounded and nondegenerate. The following lemma represents the properties that we need in the sequel.

Lemma 1. There exist positive constants $\mathrm{C}_{6}-\mathrm{C}_{12}$ such that

$$
\begin{gathered}
\sup _{X \in X_{1}} \mathbf{E} \exp \left\{(1-b)\left|X^{0}\right|\right\} \leq c_{6}, \\
c_{7} \leq \inf _{X \in X_{1} \cup X_{2}} \mathbf{E X} \leq \sup _{X \in X_{1} \cup X_{2}} \mathbf{E X} \leq c_{8}, \\
c_{9} \leq \inf _{X \in X_{1} \cup X_{2}} \operatorname{Var}(X) \leq \sup _{X \in X_{1} \cup X_{2}} \operatorname{Var}(X) \leq c_{10},
\end{gathered}
$$

(d) For each $\mathrm{x} \in(0,1)$ and each $\gamma \geq \mathrm{c}_{12}=\left(1+2 \mathrm{c}_{8}\right) \mathrm{C}_{3}^{-1}$, the inequality

$$
\mathbf{P}\left\{\gamma(\mathrm{Z})_{\gamma} \in[\mathrm{bx}, \mathrm{x}]\right\} \geq \mathrm{c}_{11} \mathrm{x}^{\alpha_{1}}
$$

is true

The "scholar" proof of Lemma 1 is omitted. We shall often combine Lemma 1 with the following scaling property of exponential families:

$$
\mathrm{a}(\lambda \mathrm{Z})_{\gamma}=\mathrm{L}(\mathrm{a} \lambda \mathrm{Z})_{\gamma / \mathrm{a}},
$$

which holds for all positive a, $\lambda, \gamma$ and r.v. Z. In particular, we have

$$
\eta_{\mathrm{j}}=\left(\lambda_{\mathrm{j}} \mathrm{Z}_{\mathrm{j}}\right)_{\gamma}={ }_{\mathrm{L}} \lambda_{\mathrm{j}}(\mathrm{Z})_{\gamma \lambda_{\mathrm{j}}} .
$$


Sometimes we use the same relation in the form

$$
\eta_{\mathrm{j}}=\gamma_{\mathrm{L}}^{-1} \cdot \gamma \lambda_{\mathrm{j}}(\mathrm{Z})_{\gamma \lambda_{\mathrm{j}}} .
$$

In particular, it follows from (4.6) and (4.7) that

$$
\left(\lambda_{\mathrm{j}}^{-1} \vee \gamma\right) \eta_{\mathrm{j}} \in \mathrm{X}_{1} \cup \mathrm{X}_{2} \text {. }
$$

LEMmA 2. Let $\gamma=\gamma(r)$ be such that the equation (3.3) holds. Let $\sigma=\sigma(\gamma)$ be defined in (2.1). Then

$$
\lim _{r \rightarrow 0} \gamma \sigma=\infty
$$

and for each $r>0$ the inequality

holds with $c_{13}=c_{7} / c_{10}^{1 / 2}$.

$$
r \geq \mathrm{c}_{13} \sigma
$$

Proof. If $\gamma \lambda_{\mathrm{j}} \geq 1$, then (4.7) yields $\gamma \eta_{\mathrm{j}}=_{\mathrm{L}} \gamma \lambda_{\mathrm{j}}(\mathrm{Z})_{\gamma \lambda_{\mathrm{j}}} \in \mathrm{X}_{1}$ and we obtain from (4.3) that

$$
\gamma^{2} \sigma^{2}=\sum \gamma^{2} \operatorname{Var}\left(\eta_{\mathrm{j}}\right) \geq \sum_{\gamma \lambda_{\mathrm{j}} \geq 1} \operatorname{Var}\left(\gamma \eta_{\mathrm{j}}\right) \geq c_{9} \#\left\{\mathrm{j} \mid \gamma \lambda_{\mathrm{j}}>1\right\} \rightarrow \infty .
$$

Similarly, (3.3), (2.1), (4.2), and (4.3) yield

$$
\begin{aligned}
\mathrm{r}^{2} / \sigma^{2} & =\left(\sum \mathbf{E} \eta_{\mathrm{j}}\right)^{2} /\left(\sum \operatorname{Var}\left(\eta_{\mathrm{j}}\right)\right) \geq \sum\left(\mathbf{E} \eta_{\mathrm{j}}\right)^{2} /\left(\sum \operatorname{Var}\left(\eta_{\mathrm{j}}\right)\right) \\
& \geq \inf \left\{\left(\mathbf{E} \eta_{\mathrm{j}}\right)^{2} / \operatorname{Var}\left(\eta_{\mathrm{j}}\right)\right\} \geq \inf _{\mathrm{X} \in \mathrm{X}_{1} \cup \mathrm{X}_{2}}\left\{(\mathbf{E X})^{2} / \operatorname{Var}(\mathrm{X})\right\} \geq \mathrm{c}_{7}^{2} / \mathrm{c}_{10}
\end{aligned}
$$

and (4.9) is verified.

The last result of this section treats again the problems of the nondegeneracy of $X_{1}$ and $X_{2}$, this time on the level of characteristic functions.

LEMma 3. (a) For any positive $\delta$ there exists $\mathrm{D}_{1}(\delta)=\mathrm{D}_{1}(\delta, \mathrm{F})$ such that for all $\mathrm{X} \in \mathrm{X}_{2}$ and $\mathrm{v} \in\left[-\mathrm{D}_{1}(\delta), \mathrm{D}_{1}(\delta)\right]$ the ch. $\mathrm{f}$. of the corresponding centered r.v. admits a representation

$$
\hat{\mathrm{X}}^{0}(\mathrm{v})=\exp \left\{-\frac{\operatorname{Var}(\mathrm{X})}{2}(1+\theta \delta) \mathrm{v}^{2}\right\}
$$

with some complex $\theta=\theta(\mathrm{X}, \mathrm{v}),|\theta| \leq 1$. Moreover, if $\mathbf{M}_{3}=\mathbf{E} \mathrm{Z}^{3}<\infty$, then a similar representation

$$
\hat{\mathrm{X}}^{0}(\mathrm{v})=\exp \left\{-\frac{\operatorname{Var}(\mathrm{X})}{2}\left(1+\theta\left\{\mathrm{M}_{3} \mathrm{C}_{9}^{-1}+\mathrm{C}_{10}^{1 / 2}\right\} \mathrm{v}\right) \mathrm{v}^{2}\right\}
$$

holds for $v \in\left[-c_{10}^{1 / 2}, c_{10}^{1 / 2}\right]$.

(b) For any $\delta \in(0,1]$ there exists a positive $\mathrm{D}_{2}(\delta)=\mathrm{D}_{2}(\delta, \mathrm{F})$ such that for each real $\mathrm{V},|\mathrm{V}|>\delta$ and each $\mathrm{X} \in \mathrm{X}_{1} \cup \mathrm{X}_{2}$,

$$
|\hat{\mathrm{X}}(\mathrm{v})| \leq 1-\mathrm{D}_{2}(\delta)|\mathrm{v}|^{-\alpha_{1}}
$$

with $\alpha_{1}$ defined in (3.1). 
REMARK. The estimate (4.11) looks bad for large values of the variable $v$ but simple examples show that nothing better can be obtained without supplementary smoothness assumptions on $F$.

Proof. (a) It follows from the classic inequality

$$
\left|\exp \{i x\}-\sum_{j=0}^{k-1}(i x)^{j} / j !\right| \leq|x|^{k} / k !
$$

(applied with $\mathrm{k}=2$ and $\mathrm{k}=3$ ) that for any centered r.v. $\mathrm{Y}$ with finite variance and any $v \in \mathbf{R}, \mathrm{D}>0$,

$$
\left|\hat{Y}(\mathrm{~V})-1+\operatorname{Var}(Y) \frac{\mathrm{v}^{2}}{2}\right| \leq\left(\frac{\mathrm{D}}{6} \operatorname{Var}(\mathrm{Y})+\mathbf{E Y}^{2} \mathbf{1}_{\{|Y|>D /|V|\}}\right) \mathrm{v}^{2} \text {. }
$$

Let now $Y=X^{0},|V| \leq D^{2}$ and $D \leq\left(E Z^{2}\right)^{-1 / 2}$. Then

$$
\begin{gathered}
\left.-\mathbf{E X} \geq-(\mathbf{E X})^{2}\right)^{1 / 2} \geq-\left(\mathbf{E} Z^{2}\right)^{1 / 2} \geq-D^{-1}, \\
Y=X-\mathbf{E X} \geq-D^{-1},
\end{gathered}
$$

$\mathbf{E Y}^{2} \mathbf{1}_{\{|\mathrm{Y}|>\mathrm{D} / \mathrm{VV} \mid\}} \leq \mathbf{E Y}^{2} \mathbf{1}_{\left\{\mathrm{Y}>\mathrm{D}^{-1}\right\}} \leq \mathbf{E} X^{2} \mathbf{1}_{\left\{\mathrm{X}>\mathrm{D}^{-1}\right\}} \leq \mathbf{E} \mathrm{Z}^{2} \mathbf{1}_{\left\{\mathrm{Z}>\mathrm{D}^{-1}\right\}}$.

Choose now $\mathrm{D}=\mathrm{D}(\delta)$ so small that $\mathrm{D}<\delta / 2$ and $\mathbf{E} \mathrm{Z}^{2} \mathbf{1}_{\left\{\mathrm{Z}>\mathrm{D}^{-1}\right\}} \leq \delta \mathrm{C}_{9} / 6$. Then, (4.12) yields the estimate

$$
\left|\hat{\mathrm{X}}^{0}(\mathrm{v})-1+\operatorname{Var}(\mathrm{X}) \mathrm{v}^{2} / 2\right| \leq \operatorname{Var}(\mathrm{X}) \delta \mathrm{v}^{2} / 4 \text {. }
$$

Moreover, if $\left.\mathrm{D} \leq(\delta \wedge 1) / \mathrm{C}_{10}\right)^{1 / 4}$, we can apply the inequality $\| \operatorname{og}(1+\mathrm{h})-\mathrm{h} \mid$ $\leq|\mathrm{h}|^{2}$ to $\mathrm{h}=\hat{\mathrm{X}}^{0}(\mathrm{v})-1$ and obtain (4.10) with $\mathrm{D}_{1}(\delta)=\mathrm{D}^{2}$. We omit the proof of (4.10a) which is essentially the same as that of (4.10).

(b) First, let $\gamma>\mathrm{C}_{12}$ and $\mathrm{X}=\gamma(\mathrm{Z})_{\gamma} \in \mathrm{X}_{1}$ (the critically difficult case). Denote $\mathrm{h}=\arg (\hat{\mathrm{X}}(\mathrm{v})) \in[0,2 \pi)$. Then

$$
1-|\hat{X}(v)|=1-\hat{X}(v) \exp \{-i h\}=\mathbf{E}(1-\cos (v X-h)) .
$$

Define four auxiliary intervals

$$
\mathrm{I}_{\mathrm{k}}=\left[\delta \mathrm{b}^{\mathrm{k}+1} /|\mathrm{v}|, \delta \mathrm{b}^{\mathrm{k}} /|\mathrm{v}|\right], \quad \mathrm{k}=0,1,2,3 .
$$

It is easy to see that for each $h \in[0,2 \pi)$ there exists $k \in[0 \ldots 3]$ such that

$$
\inf _{x \in I_{h}}[1-\cos (v x-h)] \geq\left[1-\cos \left(\delta\left(b^{3}-b^{4}\right)\right)\right]=D_{2}^{(1)}(\delta, b) .
$$

Therefore, the application of (4.4) from Lemma 1 yields

$$
\begin{aligned}
\mathbf{E}(1-\cos (\mathrm{vX}-\mathrm{h})) & \geq \mathrm{D}_{2}^{(1)} \mathbf{P}\left\{\mathrm{X} \in \mathrm{I}_{\mathrm{k}}\right\} \\
& \geq \mathrm{c}_{11} \mathrm{D}_{2}^{(1)}\left(\delta \mathrm{b}^{3} /|\mathrm{v}|\right)^{\alpha_{1}}=\mathrm{D}_{2}^{(2)}(\delta, \mathrm{b})|\mathrm{v}|^{-\alpha_{1}} .
\end{aligned}
$$

The consideration of other $\mathrm{X} \in \mathrm{X}_{1} \cup \mathrm{X}_{2}$ is easier. In fact, the mapping $\gamma \rightarrow L\left\{(Z)_{\gamma}\right\}$ is continuous in variation metric. This fact implies the continuity of the function $\gamma \rightarrow 1-\sup _{|v|>\delta}\left|(Z)_{\gamma}^{\wedge}(v)\right|$. The values of this function are all 
positive due to the absolute continuity of $L(Z)$. Hence,

$$
1-\sup _{\gamma \in\left[0, c_{12}\right]|v|>\delta} \sup _{\mid v}|(Z) \hat{\gamma}(\mathrm{v})|=\mathrm{D}_{2}^{(3)}(\delta, \mathrm{F})>0
$$

and we can put $\mathrm{D}_{2}(\delta)=\mathrm{D}_{2}^{(2)} \wedge \delta^{\alpha_{1}} \mathrm{D}_{2}^{(3)}$.

5. Zaitsev estimate in CLT. We need some appropriate tools from CLT theory in order to obtain the statements of Theorem 1. For this aim, we use a powerful and convenient approach due to A. Yu. Zaitsev $[2,16,17]$. Essential concepts of this approach are traced below.

Let $\tau>0$. Let A $(\tau)$ be the class of all r.v. $\eta$ whose characteristic functions $\phi(u)=\mathbf{E} \exp \{i u \eta\}$ satisfy the condition

$$
\left|(\log \phi)^{(3)}(\mathrm{u})\right| \leq \tau \operatorname{Var}(\eta)
$$

for each complex $\mathrm{u},|\mathrm{u}| \leq \tau^{-1}$. Parameter $\tau$ is a measure of proximity of the distribution of $\eta$ to the family of normal laws. In particular, if $\eta \in A(0)$, then $L(\eta)$ is normal. We need only a few quite elementary basic properties of classes $A(\cdot)$.

1. Each class $A(\tau)$ is closed with respect to convolution.

2. If $\tau_{1} \leq \tau_{2}$, then $\mathrm{A}\left(\tau_{1}\right) \subset \mathrm{A}\left(\tau_{2}\right)$, that is, $\mathrm{A}(\cdot)$ is monotone family.

3. If $\eta \in A(\tau), c \in R$, then $c \eta \in A(|c| \tau)$. This is a scaling property.

The single Zaitsev result which we need (actually it is available even in a multivariate setting) can be represented by the following ch.f. estimate:

$$
\log \phi(\mathrm{u})=-\frac{\operatorname{Var}(\eta)}{2} \mathrm{u}^{2}\left(1+\frac{\theta|\mathrm{u}| \tau}{3}\right)
$$

for each centered $\eta \in \mathrm{A}(\tau), \mathrm{u} \in\left[-\tau^{-1}, \tau^{-1}\right]$, with some complex $\theta,|\theta| \leq 1$.

It follows immediately from (5.2) that for each sequence $\left\{\eta_{\mathrm{n}}\right\}$ with $\mathbf{E}\left\{\eta_{\mathrm{n}}\right\}=0$, $\operatorname{Var}\left(\eta_{\mathrm{n}}\right)=1, \eta_{\mathrm{n}} \in \mathrm{A}\left(\tau_{\mathrm{n}}\right), \tau_{\mathrm{n}} \rightarrow 0$, the weak convergence of $\mathrm{L}\left(\eta_{\mathrm{n}}\right)$ to $\mathrm{N}(0,1)$ holds.

There are several possibilities in using this approach. The condition (5.1) requires the knowledge of ch.f. (which is easy to calculate, for example, in the $\chi^{2}$ case) and nothing else. On the other hand, if the information about the moments is available, we have an alternative starting point for calculations via Cramér or Bernstein conditions. We use the Cramér condition, as the simplest one for verification. Namely, combining the statements from [2], page 33, and [16], page 205, the first one concerning the relation between Cramer and Bernstein conditions, the second one concerning the relation between the Bernstein condition and $A(\cdot)$ classes, one obtains that for some absolute constant $\mathrm{C}_{14}$,

$$
\left\{\eta \mid \mathbf{E} \eta=0, \mathbf{E} \exp \left\{a_{1}|\eta|\right\} \leq a_{2}, \operatorname{Var}(\eta) \geq a_{3}\right\} \subset A\left(c_{14} a_{2} /\left(a_{1}^{3} a_{3}\right)\right)
$$

holds for all positive $a_{1}, a_{2}, a_{3}$.

The same results hold for classes of multidimensional random vectors. We do not need this extension in the current context, but it may be a useful tool 
for more sophisticated problems, for example, such as a mixed problem of "large deviations in the small ball" from [3].

\section{Proofs of main results.}

Proof of TheOREM 1. Recall that $\zeta_{\mathrm{s}}^{0}=\zeta^{0}+\varepsilon \xi / \gamma$, Our goal is to prove two following statements about the characteristic functions of $\zeta^{0}$ and $\zeta_{s}^{0}$ which imply statements (a) and (b) of Theorem 1, respectively.

(a') For each $u \in \mathbf{R}$

$$
\lim _{\gamma \rightarrow \infty} \hat{\zeta}^{0}(u / \sigma)=\exp \left\{-u^{2} / 2\right\}
$$

$\left(b^{\prime}\right)$

$$
\lim _{\gamma \rightarrow \infty} \int_{-\infty}^{\infty}\left|\hat{\zeta}_{s}^{0}(u / \sigma)-\exp \left\{-u^{2} / 2\right\}\right| d u=0 .
$$

Moreover, if $\mathbf{M}_{3}=\mathbf{E} \mathbf{Z}^{3}<\infty$, then

$$
\int_{-\infty}^{\infty}\left|\hat{\zeta}_{\mathrm{s}}^{0}(\mathrm{u} / \sigma)-\exp \left\{-\mathrm{u}^{2} / 2\right\}\right| \mathrm{du}=\mathrm{O}\left((\gamma \sigma)^{-1}\right) .
$$

First, we deal with moderate values of parameter $\mathrm{u}$. We show that for an $\gamma$ large enough and $\mathrm{u}$ such that $|\mathrm{u}| \leq \gamma \sigma$,

$$
(\varepsilon \xi / \gamma) \hat{\imath}(\mathrm{u} / \sigma)=1+\theta \mathrm{u} / \gamma \sigma,
$$

with $|\theta| \leq 1$.

Indeed, for each $\vee$ we have $|\hat{\xi}(\mathrm{v})-1| \leq \operatorname{Var} \xi \mathrm{v}^{2} / 2$. By Lemma 2 , we have $\varepsilon \rightarrow 0$ when $\gamma$ tends to infinity; hence, the inequality $|\mathrm{u}| \leq \gamma \sigma$ implies, ultimately for large $\gamma$, that

$$
|(\varepsilon \xi / \gamma) \hat{\imath}(\mathrm{u} / \sigma)-1|=|\hat{\xi}(\varepsilon \mathrm{u} / \gamma \sigma)-1| \leq|\mathrm{u}| / \gamma \sigma .
$$

This calculation proves (6.3).

Next, we show that for each fixed $\delta \in(0,1]$ there exists (small enough) positive $\mathrm{D}(\delta)$ such that for all $\gamma$ large enough and for all $\mathrm{u},|\mathrm{u}| \leq \mathrm{D}(\delta) \gamma \sigma$,

$$
\hat{\zeta}^{0}(\mathrm{u} / \sigma)=\exp \left\{-\mathrm{u}^{2}(1+\theta \delta) / 2\right\}
$$

with $|\theta| \leq 1$. If $M_{3}<\infty$, we have a stronger version of (6.4); namely, for some small $\mathrm{D}_{0}>0$ and all $\mathrm{u},|\mathrm{u}| \leq \mathrm{D}_{0} \gamma \sigma$,

$$
\hat{\zeta}^{0}(\mathrm{u} / \sigma)=\exp \left\{-\mathrm{u}^{2}\left(1+\theta \mathrm{u} / \mathrm{D}_{0} \gamma \sigma\right) / 2\right\},
$$

with $|\theta| \leq 1$.

In order to establish (6.4), let us look at the ch.f. of the terms $\eta_{\mathrm{j}}$ of the sum $\zeta$ in definition (2.1).

First, let $\gamma \lambda_{\mathrm{j}} \geq 1$. We observed in (4.7) that $\eta_{\mathrm{j}}=\mathrm{L}_{\mathrm{L}} \gamma^{-1} \mathrm{X}, \mathrm{X} \in \mathrm{X}_{1}$. Moreover, (4.1), (4.3) and (5.3) imply that $X^{0} \in A\left(c_{15}\right)$ with $c_{15}=c_{14} c_{6} /(1-b)^{3} c_{9}$. By 
the Zaitsev representation (5.2), we have

$$
\hat{\mathrm{X}}^{0}(\mathrm{v})=\exp \left\{-\frac{\operatorname{Var}(\mathrm{X})}{2} \mathrm{v}^{2}\left(1+\frac{\mathrm{c}_{15} \theta|\mathrm{v}|}{3}\right)\right\}
$$

for any $\mathrm{v}$ such that $|\mathrm{v}|<\mathrm{C}_{15}^{-1}$. We put $\mathrm{v}=\mathrm{u} / \gamma \sigma$ and see that

$$
\hat{\eta}_{\mathrm{j}}^{0}\left(\frac{\mathrm{u}}{\sigma}\right)=\hat{\mathrm{X}}^{0}\left(\frac{\mathrm{u}}{\gamma \sigma}\right)=\exp \left\{-\frac{\operatorname{Var}\left(\eta_{\mathrm{j}}\right)}{2 \sigma^{2}} \mathrm{u}^{2}\left(1+\frac{\mathrm{c}_{15} \theta|\mathrm{u}|}{3 \gamma \sigma}\right)\right\} .
$$

In particular, for $|\mathrm{u}| \leq\left(\delta / \mathrm{C}_{15}\right) \gamma \sigma$, we have the desired representation

$$
\hat{\eta}_{\mathrm{j}}^{0}\left(\frac{\mathrm{u}}{\sigma}\right)=\exp \left\{-\frac{\operatorname{Var}\left(\eta_{\mathrm{j}}\right)}{2 \sigma^{2}} \mathrm{u}^{2}\left(1+\theta_{\mathrm{j}} \delta\right)\right\} .
$$

Let now $\gamma \lambda_{\mathrm{j}} \leq 1$. In this case we use (4.6) and interpret it as

$$
\eta_{\mathrm{j}}=\lambda_{\mathrm{L}} \mathrm{X}, \quad \mathrm{X} \in \mathrm{X}_{2} .
$$

According to Lemma 3, we have

$$
\hat{X}^{0}(v)=\exp \left\{-\frac{\operatorname{Var}(\mathrm{X})}{2} \mathrm{v}^{2}(1+\theta \delta)\right\}
$$

for any $\mathrm{X} \in \mathrm{X}_{2}$ and $|\mathrm{v}|<\mathrm{D}_{1}(\delta)$. We set $\mathrm{v}=\lambda_{\mathrm{j}} \mathrm{u} / \sigma$ and observe that

$$
\hat{\eta}_{\mathrm{j}}^{0}\left(\frac{\mathrm{u}}{\sigma}\right)=\hat{\mathrm{X}}^{0}\left(\frac{\lambda_{\mathrm{j}} \mathrm{u}}{\sigma}\right)=\exp \left\{-\frac{\operatorname{Var}\left(\eta_{\mathrm{j}}\right)}{2 \sigma^{2}} \mathrm{u}^{2}\left(1+\theta_{\mathrm{j}} \delta\right)\right\}
$$

whenever $|\mathrm{u}| \leq \mathrm{D}_{1}(\delta) \gamma \sigma$ and $|\mathrm{v}| \leq \mathrm{D}_{1}(\delta) \gamma \lambda_{\mathrm{j}} \leq \mathrm{D}_{1}(\delta)$. Finally we infer from (6.5) and (6.6) that for $|\mathrm{u}| \leq\left\{\delta \mathrm{C}_{15}^{-1} \wedge \mathrm{D}_{1}(\delta)\right\} \gamma \sigma$,

$$
\hat{\zeta}^{0}(\mathrm{u} / \sigma)=\prod_{\mathrm{j}} \hat{\eta}_{\mathrm{j}}^{0}(\mathrm{u} / \sigma)=\exp \left\{-\mathrm{u}^{2}(1+\theta \delta) / 2\right\} \text {. }
$$

Thus, the representation (6.4) is proved.

Under assumption $\mathrm{M}_{3}<\infty$, we may use the stronger version of Lemma 3 and apply (4.10a). Then, for the case $\gamma \lambda_{\mathrm{j}} \leq 1,|\mathrm{u}| \leq \mathrm{C}_{10}^{-1 / 2} \gamma \sigma$, we have

$$
\begin{aligned}
\hat{\eta}_{\mathrm{j}}^{0}\left(\frac{\mathrm{u}}{\sigma}\right) & =\hat{\mathrm{X}}^{0}\left(\frac{\lambda_{\mathrm{j}} \mathrm{u}}{\sigma}\right) \\
& =\exp \left\{-\frac{\operatorname{Var}\left(\eta_{\mathrm{j}}\right)}{2 \sigma^{2}} \mathrm{u}^{2}\left(1+\theta_{\mathrm{j}}\left(\mathrm{M}_{3} \mathrm{C}_{9}^{-1}+\mathrm{c}_{10}^{1 / 2}\right) \frac{\mathrm{u}}{\gamma \sigma}\right)\right\} .
\end{aligned}
$$

By the old arguments, we obtain now for $|\mathrm{u}| \leq\left\{\mathrm{C}_{15}^{-1} \wedge \mathrm{C}_{10}^{-1 / 2}\right\} \gamma \sigma$,

$$
\hat{\zeta}^{0}(\mathrm{u} / \sigma)=\exp \left\{-\mathrm{u}^{2}\left(1+\theta \mathrm{u} / \mathrm{D}_{0} \gamma \sigma\right) / 2\right\},
$$

if $D_{0}^{-1} \geq\left(M_{3} C_{9}^{-1}+c_{10}^{1 / 2}\right)$ and $D_{0}^{-1} \geq C_{15} / 3$. Thus, the representation (6.4a) is also proved. 
It is plain that (6.4) implies the CLT statement (6.1). On the other hand, the identity $\hat{\zeta}_{s}^{0}=\hat{\zeta}^{0} \cdot(\varepsilon \xi / \gamma)^{\wedge}$ combined with (6.3), (6.4) and with elementary estimate $|\exp (x)-1| \leq|x| \exp (|x|)$ easily yields

$$
\begin{gathered}
\int_{|\mathrm{u}|<\{\mathrm{D}(\delta) \wedge 1\} \gamma \sigma}\left|\hat{\zeta}_{\mathrm{s}}^{0}(\mathrm{u} / \sigma)-\exp \left\{-\mathrm{u}^{2} / 2\right\}\right| \mathrm{du} \\
\leq(2 \pi)^{1 / 2}(1-\delta)^{-3 / 2} \delta+2(\gamma \sigma)^{-1}
\end{gathered}
$$

Similarly, if $\mathrm{M}_{3}<\infty$, we use (6.4a) instead of (6.4) and obtain

$$
\int_{|\mathrm{u}|<\left\{1 \wedge \mathrm{D}_{0} / 2\right\} \gamma \sigma}\left|\hat{\zeta}_{\mathrm{s}}^{0}(\mathrm{u} / \sigma)-\exp \left\{-\mathrm{u}^{2} / 2\right\}\right| \mathrm{du} \leq\left(16 \mathrm{D}_{0}^{-1}+2\right)(\gamma \sigma)^{-1} .
$$

Thus, (6.2) and (6.2a) boil down to the demonstration of the tail estimate for ch.f.:

$$
\int_{|\mathrm{u}| \geq \mathrm{D} \gamma \sigma}\left|\hat{\zeta}_{\mathrm{s}}^{0}(\mathrm{u} / \sigma)\right| \mathrm{du}=\mathrm{O}\left((\gamma \sigma)^{-1}\right)
$$

for each $D>0$, and we can from now concentrate our considerations on the proof of this estimate. For this aim, we introduce two index sets, $J_{1}=\{j \mid \gamma \vee$ $\left.\left.\lambda_{\mathrm{j}}^{-1} \geq \mathrm{m}\right\}, \mathrm{J}_{2}=\mathrm{j} \mid \gamma \vee \lambda_{\mathrm{j}}^{-1} \leq \mathrm{m}\right\}$, and choose the median $\mathrm{m}=\mathrm{m}(\gamma)$ as the unique integer value providing both inequalities:

$$
\sum_{J_{1}} \sigma_{\mathrm{j}}^{2} \geq \sigma^{2} / 2, \quad \sum_{\mathrm{J}_{2}} \sigma_{\mathrm{j}}^{2} \geq \sigma^{2} / 2
$$

Put $C_{16}=\min \left\{C_{15}^{-1}, D_{1}(1 / 3)\right\}$, where $D_{1}(\cdot)$ was defined in statement (a) of Lemma 3. The crucial point is now to show that for each $\mathrm{j} \in \mathrm{J}_{1}$ and each $u$ such that $|\mathrm{u}| \leq \mathrm{c}_{16} \mathrm{~m} \sigma$, the estimate

$$
\left|\hat{\eta}_{\mathrm{j}}^{0}\left(\frac{\mathrm{u}}{\sigma}\right)\right| \leq \exp \left\{\frac{-\sigma_{\mathrm{j}}^{2} \mathrm{u}^{2}}{3 \sigma^{2}}\right\}
$$

holds. Provided that this is true, we have immediately

$$
\left|\hat{\zeta}_{\mathrm{s}}^{0}\left(\frac{\mathrm{u}}{\sigma}\right)\right| \leq \prod_{\mathrm{j}}\left|\hat{\eta}_{\mathrm{j}}^{0}\left(\frac{\mathrm{u}}{\sigma}\right)\right| \leq \exp \left\{-\sum_{\mathrm{J}_{1}} \frac{\sigma_{\mathrm{j}}^{2} \mathrm{u}^{2}}{3 \sigma^{2}}\right\} \leq \exp \left\{\frac{-\mathrm{u}^{2}}{6}\right\}
$$

and, using Lemma 2,

$$
\begin{aligned}
\int_{\mathrm{D} \gamma \sigma \leq|\mathrm{u}| \leq \mathrm{c}_{16} \mathrm{~m} \sigma}\left|\hat{\zeta}_{\mathrm{s}}^{0}(\mathrm{u} / \sigma)\right| \mathrm{du} & \leq \int_{\mathrm{D} \gamma \sigma \leq|\mathrm{u}|} \exp \left\{\frac{-\mathrm{u}^{2}}{6}\right\} \mathrm{du} \\
& =\mathrm{O}\left(\exp \left\{-\frac{\mathrm{D}^{2} \gamma^{2} \sigma^{2}}{2}\right\}\right) .
\end{aligned}
$$

Consider now two separate cases in order to obtain (6.9).

(a) $\gamma \lambda_{\mathrm{j}} \geq 1$. We have al ready deduced from (4.7) that

$$
\eta_{\mathrm{j}}^{0}=\gamma_{\mathrm{L}} \gamma^{-1} \mathrm{X}^{0}, \quad \mathrm{X}^{0} \in \mathrm{A}\left(\mathrm{C}_{15}\right) \text {. }
$$


Hence, $\eta_{\mathrm{j}}^{0} \in \mathrm{A}\left(\mathrm{c}_{15} / \gamma\right)$. Notice also that in the considered domain,

$$
|\mathrm{u}| / \sigma \leq \mathrm{c}_{16} \mathrm{~m} \leq \mathrm{c}_{16}\left(\gamma \vee \lambda_{\mathrm{j}}^{-1}\right)=\mathrm{c}_{16} \gamma \leq \gamma / \mathrm{c}_{15} .
$$

Therefore, the Zaitsev estimate (5.2) yields

$$
\left|\hat{\eta}_{\mathrm{j}}^{0}(\mathrm{u} / \sigma)\right| \leq \exp \left\{\frac{-\sigma_{\mathrm{j}}^{2} \mathrm{u}^{2}}{2 \sigma^{2}}\left(1-\frac{\mathrm{c}_{15}|\mathrm{u}|}{3 \gamma \sigma}\right)\right\} \leq \exp \left\{-\frac{\sigma_{\mathrm{j}}^{2} \mathrm{u}^{2}}{3 \sigma^{2}}\right\} .
$$

(b) $\gamma \lambda_{\mathrm{j}} \leq 1$. Making use of decomposition (4.6), we obtain

and

$$
\hat{\eta}_{\mathrm{j}}^{0}(\mathrm{u} / \sigma)=\hat{\mathrm{X}}^{0}\left(\mathrm{u} \lambda_{\mathrm{j}} / \sigma\right), \quad \mathrm{X} \in \mathrm{X}_{2},
$$

$$
|\mathrm{u}| \lambda_{\mathrm{j}} / \sigma \leq \mathrm{c}_{16} \mathrm{~m} \lambda_{\mathrm{j}} \leq \mathrm{c}_{16}\left(\gamma \vee \lambda_{\mathrm{j}}^{-1}\right) \lambda_{\mathrm{j}}=\mathrm{c}_{16} \leq \mathrm{D}_{1}(1 / 3) .
$$

Now the direct application of (4.10) proves (6.9) and (6.10).

Next, consider the integration domain $\mathrm{U}=\left\{\mathrm{u} \in \mathbf{R}, \quad \mathrm{c}_{16} \mathrm{~m} \sigma \leq|\mathrm{u}| \leq\right.$ $\left.\varepsilon^{-(1+\kappa / 2)} \gamma \sigma\right\}$. We can apply here the chain of estimates

$$
\left|\hat{\zeta}_{\mathrm{s}}^{0}(\mathrm{u} / \sigma)\right| \leq \prod_{\mathrm{j}_{2}}\left|\hat{\eta}_{\mathrm{j}}^{0}(\mathrm{u} / \sigma)\right|=\prod_{\mathrm{j}_{2}}\left|\hat{\mathrm{X}}_{\mathrm{j}}^{0}\left(\left(\gamma^{-1} \wedge \lambda_{\mathrm{j}}\right) \mathrm{u} / \sigma\right)\right|
$$

with some $\mathrm{X}_{\mathrm{j}} \in \mathrm{X}_{1} \cup \mathrm{X}_{2}$. Observe that for $\mathrm{u} \in \mathrm{U}, \mathrm{j} \in \mathrm{J}_{2}$ we have

$$
\begin{aligned}
\varepsilon^{-(1+\kappa / 2)} & \geq \varepsilon^{-(1+\kappa / 2)} \gamma \sigma\left(\gamma^{-1} \wedge \lambda_{\mathrm{j}}\right) / \sigma \geq\left(\gamma^{-1} \wedge \lambda_{\mathrm{j}}\right)|\mathrm{u}| / \sigma \\
& \geq \mathrm{c}_{16} \mathrm{~m} /\left(\gamma \vee \lambda_{\mathrm{j}}^{-1}\right) \geq \mathrm{c}_{16} .
\end{aligned}
$$

Thus, we can apply Lemma 3 and (4.11) yields the estimate

$$
\left|\hat{\zeta}_{\mathrm{s}}^{0}(\mathrm{u} / \sigma)\right| \leq \prod_{\mathrm{J}_{2}}\left(1-\mathrm{C}_{17} \varepsilon^{(1+\kappa / 2) \alpha_{1}}\right) \leq \exp \left\{-\mathrm{C}_{17} \#\left\{\mathrm{j} \in \mathrm{J}_{2}\right\} \varepsilon^{(1+\kappa / 2) \alpha_{1}}\right\}
$$

with $c_{17}=D_{2}\left(c_{16} \wedge 1\right)$ where the function $D_{2}$ was defined in (4.11).

The number of integers in $\mathrm{J}_{2}$ also can be estimated:

$$
\begin{aligned}
\sigma^{2} / 2 & \leq \sum_{\mathrm{J}_{2}} \sigma_{\mathrm{j}}^{2} \leq \#\left\{\mathrm{j} \in \mathrm{J}_{2}\right\} \sup _{\mathrm{J}_{2}} \sigma_{\mathrm{j}}^{2} \leq \#\left\{\mathrm{j} \in \mathrm{J}_{2}\right\} \sup _{\mathrm{X}_{1} \cup \mathrm{X}_{2}} \operatorname{Var}(\mathrm{X}) / \gamma^{2} \\
& \leq \#\left\{\mathrm{j} \in \mathrm{J}_{2}\right\} \mathrm{C}_{10} / \gamma^{2} .
\end{aligned}
$$

Finally, by the definition of $\varepsilon$ and (4.8), we have

$$
\begin{aligned}
\int_{U}\left|\hat{\zeta}_{\mathrm{s}}^{0}(\mathrm{u} / \sigma)\right| \mathrm{du} & \leq 2 \varepsilon^{-(1+\kappa / 2)} \gamma \sigma \exp \left\{-\mathrm{c}_{17} \gamma^{2} \sigma^{2} \varepsilon^{(1+\kappa / 2) \alpha_{1}} / 2 \mathrm{c}_{10}\right\} \\
& \leq 2 \varepsilon^{-(1+\kappa / 2)} \gamma \sigma \exp \left\{-\mathrm{c}_{17}(\gamma \sigma)^{\kappa^{2} / 2} / 2 \mathrm{c}_{10}\right\} \\
& =\mathrm{O}\left((\gamma \sigma)^{-1}\right) .
\end{aligned}
$$

For the last domain $\mathrm{U}^{\prime}=\left\{\mathrm{u} \in \mathbf{R},|\mathrm{u}| \geq \varepsilon^{-(1+\kappa / 2)} \gamma \sigma\right\}$ we apply a smoothing argument. Namely, we use the trivial estimate

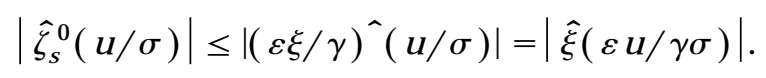


Since the characteristic function of smooth compact distribution decreases faster than any polynomial of negative degree, we have for any $n \geq 1$,

$$
\int_{\mathrm{U}^{\prime}}|\hat{\xi}(\varepsilon \mathrm{u} / \gamma \sigma)| \mathrm{du}=\gamma \sigma \varepsilon^{-1} \int_{|\mathrm{v}| \geq \varepsilon^{-1}}|\hat{\xi}(\mathrm{v})| \mathrm{du}=\gamma \sigma \varepsilon^{-1} \mathrm{o}\left(\varepsilon^{\mathrm{n}}\right) \text {. }
$$

This quantity is $\mathrm{O}\left((\gamma \sigma)^{-1}\right)$ if $\mathrm{n}>1+2 \alpha_{1} /(2-\kappa)$.

Now the statement (6.8) is proved completely and the statements of Theorem 1 follow from (6.1), (6.2) and (6.2a).

Proof of Theorem 2. Recall that $p, q, q_{0}, q_{0 s}$ are the densities of r.v. S, $\zeta, \zeta^{0} / \sigma$ and $\zeta^{0} / \sigma+(\varepsilon \xi / \gamma \sigma)$, respectively, while index zero stands for centering and index $s$ denotes smoothing. By the definition of $\mathrm{p}, \zeta$ and $\Lambda$, we have

$$
q(u)=p(u) \exp \{-\gamma u\} / \Lambda(\gamma)
$$

and

$$
\begin{aligned}
\mathbf{P}\{\mathrm{S} \leq \mathrm{r}\} & =\int_{-\infty}^{\mathrm{r}} \mathrm{p}(\mathrm{u}) \mathrm{du}=\Lambda(\gamma) \int_{-\infty}^{r} \exp \{\gamma \mathrm{u}\} \mathrm{q}(\mathrm{u}) \mathrm{du} \\
& =\Lambda(\gamma) \int_{-\infty}^{r} \exp \{\gamma \mathrm{u}\} \mathrm{q}_{0}\left(\frac{\mathrm{u}-\mathrm{r}}{\sigma}\right) \frac{\mathrm{du}}{\sigma} \\
& =\Lambda(\gamma) \exp \{\gamma \mathrm{r}\} \int_{-\infty}^{0} \exp \{\gamma \sigma \mathrm{v}\} \mathrm{q}_{0}(\mathrm{v}) \mathrm{dv} .
\end{aligned}
$$

In order to verify (3.4) and (3.4a), we need now only to check the equivalencies

$$
\int_{-\infty}^{0} \exp \{\gamma \sigma \mathrm{v}\} \mathrm{q}_{0}(\mathrm{v}) \mathrm{dv}=(2 \pi)^{-1 / 2}(\gamma \sigma)^{-1}(1+\mathrm{o}(1))
$$

and

$$
\begin{aligned}
\int_{-\infty}^{0} \exp \{\gamma \sigma \mathrm{v}\} \mathrm{q}_{0}(\mathrm{v}) \mathrm{dv} \\
\quad=(2 \pi)^{-1 / 2}(\gamma \sigma)^{-1}\left(1+\mathrm{O}\left((\gamma \sigma)^{-1}\right)+\mathrm{O}(\varepsilon)\right),
\end{aligned}
$$

respectively. Let $\mathrm{X}=\zeta^{0} / \sigma, \varepsilon=(\gamma \sigma)^{-(2-\kappa) / \alpha_{1}}, \mathrm{Y}=(\varepsilon \xi / \gamma \sigma)$. In view of the boundedness of $\xi$, the estimate $|\mathrm{Y}| \leq \varepsilon / \gamma \sigma$ is true and we can rewrite and treat the integral (6.14) as follows.

$$
\begin{aligned}
\mathbf{E} \exp \{\gamma \sigma \mathrm{X}\} \mathbf{1}_{\{\mathrm{X} \leq 0\}} & \leq \mathbf{E} \exp \{\gamma \sigma \mathrm{X}\} \mathbf{1}_{\{\mathrm{X}+\mathrm{Y} \leq \varepsilon / \gamma \sigma\}} \exp \{\gamma \sigma \mathrm{Y}+\varepsilon\} \\
& =\mathbf{E} \exp \{\gamma \sigma(\mathrm{X}+\mathrm{Y})\} \mathbf{1}_{\{\mathrm{X}+\mathrm{Y} \leq \varepsilon / \gamma \sigma\}} \exp \{\varepsilon\} \\
& =\exp \{\varepsilon\} \int_{-\infty}^{\varepsilon / \gamma \sigma} \exp \{\gamma \sigma \mathrm{v}\} \mathrm{q}_{0 \mathrm{~s}}(\mathrm{v}) \mathrm{d} \mathrm{v} .
\end{aligned}
$$


The similar lower bound also holds:

$$
\mathbf{E} \exp \{\gamma \sigma \mathrm{X}\} \mathbf{1}_{\{\mathrm{X} \leq 0\}} \geq \exp \{-\varepsilon\} \int_{-\infty}^{-\varepsilon / \gamma \sigma} \exp \{\gamma \sigma \mathrm{v}\} \mathrm{q}_{\mathrm{os}}(\mathrm{v}) \mathrm{dv} \text {. }
$$

Thus, we have reduced the problem to the investigation of smoothed distributions. By statement (b) of Theorem 1 we have

$$
\sup _{v \in[-\infty, \varepsilon / \gamma \sigma]}\left|q_{0 s}(v)-(2 \pi)^{-1 / 2} \exp \left\{-v^{2} / 2\right\}\right|=o(1)
$$

and

$$
\begin{aligned}
& \int_{-\infty}^{\varepsilon / \gamma \sigma} \exp \{\gamma \sigma \mathrm{v}\} \mathrm{q}_{0 \mathrm{~s}}(\mathrm{v}) \mathrm{dv} \\
& \leq \int_{-\infty}^{\varepsilon / \gamma \sigma} \exp \{\gamma \sigma \mathrm{v}\} \mathrm{dv} \\
& \quad \times\left((2 \pi)^{-1 / 2}+\sup _{\mathrm{v}}\left|\mathrm{q}_{0 \mathrm{~s}}(\mathrm{v})-(2 \pi)^{-1 / 2} \exp \left\{-\mathrm{v}^{2} / 2\right\}\right|\right) \\
& =\exp \{\varepsilon\}(\gamma \sigma)^{-1}\left((2 \pi)^{-1 / 2}+\mathrm{o}(1)\right) \\
& =(\gamma \sigma)^{-1}(2 \pi)^{-1 / 2}(1+\mathrm{o}(1)+\mathrm{O}(\varepsilon)) .
\end{aligned}
$$

The corresponding lower bound holds by the same reasons. Therefore, we have verified that (6.14) is true. One obtains (6.14a) from (3.2a) by the same reasoning.

Sometimes the following extension of Theorem 2 may be useful. Given $x=x(\gamma)=o(\gamma \sigma)$, let $r_{x}=r+x / \gamma$. Then, by virtue of arguments used in the proof of Theorem 2, we can obtain for $r \rightarrow 0$ that

$$
\begin{aligned}
\mathbf{P}\{\mathrm{S} \leq & \left.\mathrm{r}_{\mathrm{x}}\right\} \\
= & (2 \pi)^{-1 / 2}(\gamma \sigma)^{-1} \exp \left\{\gamma \mathrm{r}_{\mathrm{x}}\right\} \\
& \times \Lambda(\gamma)\left[1+\mathrm{O}\left((\gamma \sigma)^{-1}+(\gamma \sigma)^{-(2-\kappa) / \alpha_{1}}+(\gamma \sigma)^{-2} \mathrm{x}^{2}\right)\right] .
\end{aligned}
$$

This relation boils down to (3.4) when $x=0$. It also enables substituting the restrictive equality assumption (3.3) in Theorem 2 by the weaker approximate relation (3.5). Indeed, provided (3.5) holds, we may apply (6.18) with $\mathbf{X}=\gamma\left[\mathrm{r}-\mathbf{E}(\mathrm{S})_{\gamma}\right]$ and after trivial variable change we obtain for $r \rightarrow 0$,

$$
\begin{aligned}
\mathbf{P}\{\mathrm{S} \leq \mathrm{r}\}= & (2 \pi)^{-1 / 2}(\gamma \sigma)^{-1} \exp \{\gamma \mathrm{r}\} \\
& \times \Lambda(\gamma)\left[1+\mathrm{O}\left((\gamma \sigma)^{-1}+(\gamma \sigma)^{-(2-\kappa) / \alpha_{1}}\right.\right. \\
& \left.\left.+\sigma^{-2}\left(\mathrm{r}-\mathbf{E}(\mathrm{S})_{\gamma}\right)^{2}\right)\right] .
\end{aligned}
$$


Proof of TheOREM 3. In order to obtain the local limit statement (a) of Theorem 3, it suffices to prove that

$$
\lim _{\gamma \rightarrow \infty} \int_{-\infty}^{\infty}\left|\hat{\zeta}^{0}(u / \sigma)-\exp \left\{-u^{2} / 2\right\}\right| d u=0,
$$

which is equivalent, up to smoothing of $\zeta$, to the statement (6.2). Therefore, we can simply repeat the arguments of the proof of (6.2) with $\xi=0$, that is, without smoothing. The single difficulty that we must avoid is the estimate of the integral over high-frequency domain,

$$
\int_{\mathrm{U}^{\prime}}\left|\hat{\zeta}_{0}(\mathrm{u} / \sigma)\right| \mathrm{du}, \quad \mathrm{U}^{\prime}=\left\{\mathrm{u} \in \mathbf{R},|\mathrm{u}| \geq \varepsilon^{-(1+\kappa / 2)} \gamma \sigma\right\},
$$

which was previously treated by a smoothing argument in (6.12). Now we replace it by another reasoning based on supplementary assumptions (3.6) and (3.7). Namely, by the definition of $\zeta$ and $\varepsilon$, we can write

$$
\int_{\mathrm{U}^{\prime}}\left|\hat{\zeta}_{0}(\mathrm{u} / \sigma)\right| \mathrm{du} \leq \gamma \sigma \int_{|\mathrm{v}| \geq(\gamma \sigma)^{\left(2-\kappa^{2} / 2\right) / \alpha_{1}}} \prod_{\mathrm{j}=1}^{\mathrm{n}}\left|\hat{\mathrm{X}}_{\mathrm{j}}(\mathrm{v})\right| \mathrm{dv},
$$

where $\mathrm{n}$ will be a large but fixed integer, and $\mathrm{X}_{\mathrm{j}}=\gamma \eta_{\mathrm{j}} \in \mathrm{X}_{1}$, whenever $\gamma \lambda_{\mathrm{j}} \geq 1$. Moreover, making use of (3.6) and (3.7), we can derive for each $\mathrm{j} a$ decomposition

$$
\hat{\mathrm{X}}_{\mathrm{j}}(\mathrm{v})=\hat{\mathrm{X}}_{\mathrm{j} 0}(\mathrm{v})+\hat{\mathrm{X}}_{\mathrm{j} 1}(\mathrm{v}) \text {, }
$$

such that smoothness assumption (3.6) yields the polynomial decrease of $\hat{X}_{j 0}$; that is,

$$
\sup _{\mathrm{j}}\left|\hat{\mathrm{X}}_{\mathrm{j} 0}(\mathrm{v})\right| \leq \mathrm{C}_{0}^{\prime}|\mathrm{v}|^{-\alpha}, \quad|\mathrm{v}| \geq \mathrm{v}_{0},
$$

with $\mathrm{C}_{0}^{\prime}$ and $\alpha$ depending only on $\mathrm{C}$ and $\alpha_{1}$. On the other hand, each $\hat{\mathrm{X}}_{\mathrm{j} 1}$ turns out to be Fourier transform of some positive function $f_{1 j}$ such that $\|\mathrm{f}\|_{1} \leq 1$. Moreover, we have, due to assumption (3.7),

$$
\sup _{j} \int_{-\infty}^{\infty} f_{1 j}(x)^{p} d x \leq C_{1}^{\prime} \int_{K}^{\infty} f(x)^{p} d x e^{-p K \gamma} \gamma^{p \alpha_{1}}<\infty .
$$

We may assume, without loss of generality, that $p \leq 2$. In this case we can apply a classic Titchmarsch theorem ([15], Section 4) extending Parseval identity and thus obtain (omitting the details) for adjoint index $p^{\prime}=p /(p-$ $1)$,

$$
\sup _{j} \int_{-\infty}^{\infty}\left|\hat{X}_{1 j}(v)\right|^{p^{\prime}} d v \leq C_{2}^{\prime}\left\{\int_{K}^{\infty} f(x)^{p} d x\right\}^{p^{\prime} / p} \exp \left(-p^{\prime} K \gamma \lambda_{n}\right) \gamma^{p^{\prime} \alpha_{1}}
$$

with $\mathrm{C}_{2}^{\prime}$ depending only on $\mathrm{F}, \mathrm{p}$.

Finally, the simple combination of (6.21) and (6.22) shows that the integral (6.20) vanishes when $\gamma$ tends to infinity. Thus, statement (a) of Theorem 3 is established. 
Statement (b) of this theorem follows immediately from statement (a) and identity (6.13), that is,

$$
p(r)=q(r) \exp \{\gamma r\} \Lambda(\gamma)=q_{0}(0) \sigma^{-1} \exp \{\gamma r\} \Lambda(\gamma),
$$

where $\mathrm{p}, \mathrm{q}, \mathrm{q}_{0}$ are the densities of r.v. $\mathrm{S}, \zeta, \xi_{0}$, respectively, and, as we have just proved, $\lim _{\gamma \rightarrow \infty} q_{0}(0)=(2 \pi)^{-1 / 2}$.

Proof of TheOREM 4. We only give an idea of the proof, which follows the scheme used in Theorems 1 and 2, but is easier, since the explicit formulas for ch.f. are known. Namely, for each fixed t, we must investigate the lower tail probabilities of r.v. $\mathrm{S}=\sum\left(\lambda_{\mathrm{j}}^{1 / 2} \xi_{\mathrm{j}}-\alpha_{\mathrm{j}}\right)^{2}$. It is easy to see that

$$
(\mathrm{S})_{\gamma}=\mathrm{L} \sum\left(\beta_{\mathrm{j}}^{1 / 2} \xi_{\mathrm{j}}-\omega_{\mathrm{j}}\right)^{2}
$$

with $\beta_{\mathrm{j}}=\lambda_{\mathrm{j}}\left(1+2 \gamma \lambda_{\mathrm{j}}\right)^{-1}, \quad \omega_{\mathrm{j}}=\alpha_{\mathrm{j}}\left(1+2 \gamma \lambda_{\mathrm{j}}\right)^{-1}$. The calculation of $\chi^{2}$-characteristic functions shows that for each $\beta>0, \omega \in \mathbf{R}$ and standard normal r.v. $\xi$, we have

$$
\left(\beta^{1 / 2} \xi-\omega\right)^{2} \in A(k \beta),
$$

where $A(\cdot)$ is the Zaitsev class introduced in Section 5 and $k$ is an absolute constant. It is remarkable that the same value of parameter appears for all real $\omega$. The scaling properties of Zaitsev classes yield

$$
(\mathrm{S})_{\gamma} \in \mathrm{A}\left(\mathrm{ksup}_{\mathrm{j}} \beta_{\mathrm{j}}\right) \subset \mathrm{A}\left(2 \mathrm{k} \gamma^{-1}\right) \text {. }
$$

Moreover, we infer from (3.8) that

$$
\sigma^{-1}(\mathrm{~S})_{\gamma} \in \mathrm{A}\left(2 \mathrm{k}(\sigma \gamma)^{-1}\right) \searrow \mathrm{A}(0),
$$

which means that the central limit theorem holds for $(S)_{\gamma}$ and that the corresponding estimates of convergence rates of characteristic functions are valid (cf. the proof of Theorem 2). In this case we do not need smoothing procedures, since $\chi^{2}$-characteristic functions decrease with known (polynomial) rate at infinity. The passage from the CLT for $(S)_{\gamma}$ to the final asymptotics (3.10) is the same as in the proof of Theorem 2.

Acknowledgments. It is a great pleasure for the author to thank colleagues from the Mathematics Department of L. Pasteur University (Strasbourg) for their hospitality and also Th. Dunker, X. Fernique, W. Linde and A. Zaitsev for useful and "just in time" comments concerning this article. Some presentation improvements are due to a referee's advice.

\section{REFERENCES}

[1] Albin, P. (1994). Minima of H-valued Gaussian processes. Unpublished manuscript.

[2] Arak, T. V. and ZaitseV, A. Yu. (1988). Uniform limit theorems for sums of independent random variables. Proc. Steklov Inst. Math. 174 1-220.

[3] Ben Arous, G., Gardinaru, M. and Ledoux, M. (1994). Hölder norms and the support theorem for diffusions. Ann. Inst. H. Poincaré 30 415-436. 
[4] DAVIS, R. and RESNICK, S. (1991). Extremes of moving averages of random variables with finite endpoint. Ann. Probab. 19 312-328.

[5] Dembo, A., Mayer-Wolf, E. and Zeituni, O. (1995). Exact behavior of Gaussian seminorms. Probab. Statist. Letters 23 275-280.

[6] Hoffman-J orgensen, J ., Shepp, A. and Dudley, R. M. (1979). On the lower tail of Gaussian seminorms. Ann. Probab. 7 319-342.

[7] IbRagimov, I. A. (1982). On the probability that a Gaussian vector with values in a Hilbert space hits a sphere of small radius. J ournal of Soviet Mathematics $202164-2174$.

[8] Kuelbs, J. and LI, W. V. (1993). Metric entropy and the small ball problem for Gaussian measures. J. Funct. Anal. 116 133-157.

[9] Kuelbs, J., LI, W. V. and LINDE, W. (1994). The Gaussian measure of shifted balls. Probab. Theory Related Fields 98 143-162.

[10] LI, W. V. (1992). On the lower tail of Gaussian measures. In Probability in Banach Spaces 8 106-117. Birkhäuser, Boston.

[11] LI, W. V. and LINDE, W. (1993). Small ball problems for non-centered Gaussian measures. Probability and Mathematical Statistics 14 231-251.

[12] LIFSHITS, M. A. (1994). Investigation of tail behavior of Gaussian suprema by means of Laplace transform. Ann. Inst. H. Poincaré 30 163-179.

[13] Mayer-Wolf, E. and Zeitouni, O. (1993). The probability of small Gaussian ellipsoids and associated conditional moments. Ann. Probab. 21 14-24.

[14] Sytaya, G. N. (1974). On some asymptotic representation of the Gaussian measure in a Hilbert space. Theory of Stochastic Processes 2 94-104.

[15] Titchmarsch, E. C. (1948). Introduction to the Theory of Fourier Integrals. Oxford Univ. Press.

[16] ZaITSEV, A. Yu. (1987). Estimates of the Levy-Prokhorov distance in the multivariate central limit theorem for random variables with finite exponential moments. Theory Probab. Appl. 31 203-220.

[17] ZaItSEV, A. Yu. (1987). On the Gaussian approximation of convolutions under multidimensional analogues of S. N. Bernstein's inequality conditions. Probab. Theory Related Fields 74 535-566.

[18] Zolotarev, V. M. (1986). Asymptotic behavior of the Gaussian measure in $\mathrm{I}_{2}$. J ournal of Soviet Mathematics 24 2330-2334.

[19] Zolotarev, V. M. (1979). Gaussian measure asymptotic in $I_{p}$ on a set of centered sphere with radii tending to zero. Proceedings Twelfth European Meeting of Statisticians, Varna 254.

KOMENDANTSKII PROSPECT , 22-2-49

St. Petersburg 197372

RUSSIA

E-MAIL: mikhail@lifshits.spb.su 